\title{
Comparison of long-term outcomes after directional versus rotational atherectomy in peripheral artery disease
}

\author{
Adam Janas ${ }^{1}$, Krzysztof Milewski ${ }^{1}$, Piotr Buszman ${ }^{1}$, Aleksandra Kolarczyk-Haczyk ${ }^{1}$, Wojciech Trendel ${ }^{1}$, \\ Maciej Pruski ${ }^{1}$, Wojciech Wojakowski², Paweł Buszman ${ }^{1}$, Radosław S. Kiesz ${ }^{3}$ \\ ${ }^{1}$ Center of Cardiovascular Research and Development, American Heart of Poland, Katowice, Poland \\ ${ }^{2}$ Third Division of Cardiology, Medical University of Silesia, Katowice, Poland \\ ${ }^{3}$ San Antonio Endovascular \& Heart Institute, San Antonio, USA
}

Adv Interv Cardiol 2020; 16, 1 (59): 76-81

DOI: https://doi.org/10.5114/aic.2020.93914

\begin{abstract}
A bstract
Introduction: The rate of atherectomy utilization in peripheral artery diseases (PAD) is growing. The two atherectomy devices available on the market and used most frequently are the directional and rotational ones. Nonetheless, there is a lack of direct comparison between these two types of atherectomy in PAD.

Aim: To compare the long-term outcomes after PAD endovascular revascularization with two types of atherectomies: rotational (AR) (Phoenix Philips) and directional (AD) (SilverHawk Medtronic).

Material and methods: This was a single-center, retrospective study of obstructive and symptomatic PAD patients who underwent revascularization with atherectomy. The endpoints were considered as target lesion revascularization (TLR), death, amputations and bailout stenting (BS).

Results: The AR group consisted of 97 patients, while the AD group consisted of 85 individuals. There were no significant differences between the groups in terms of baseline characteristics except for an increased critical limb ischemia (CLI) prevalence in the AR group. The mean follow-up for AD and AR was $282.6 \pm 147.4$ and $255.7 \pm 186.1$ days, respectively $(p=0.44)$. There were no significant differences in the death rate (AD: $1(1.7 \%)$ vs. AR: $5(5.7 \%) ; p=0.54)$, amputations (AD: $2(2.3 \%)$ vs. AR: 5 (5.7\%); $p=0.45)$ or bailout stenting (AD: $2(2.3 \%)$ vs. AR: $3(3.2 \%) ; p=0.74)$, whereas TLR was more frequent in the AD group (AD: 25 (29\%) vs. AR: 15 (15.9\%; $p=0.03)$. The Kaplan-Meier analysis showed no significant differences between the groups in time to TLR, amputation or death.

Conclusions: In this hypothesis-generating study the AR had a lower rate of TLR when compared to the AD. Nevertheless, this
\end{abstract} should be confirmed in further controlled randomized trials.

Key words: peripheral arterial disease, critical limb ischemia, atherectomy, claudication.

S u m m a ry

The rate of atherectomy utilization in peripheral artery diseases growing. The two atherectomy devices available on the market and used most frequently are the directional and rotational ones. The aim of the study is to compare the long-term outcomes after peripheral artery diseases (PAD) revascularization with directional and rotational atherectomies. This was a single-center, retrospective study of obstructive and symptomatic PAD patients who underwent revascularization with atherectomy. There were no differences between groups in bailout stenting, amputations or death rate. Target lesion revascularization (TLR) was more frequent in the directional atherectomy group. In this hypothesis-generating study of patients with symptomatic PAD, revascularization with rotational atherectomy had a lower rate of TLR when compared to the directional one.

\section{Introduction}

The rate of the peripheral arterial disease (PAD) has been constantly growing. Four percent of the adult pop- ulation in the US will develop claudication in middle age and approximately $14 \%$ after the age of $70[1,2]$. Simultaneously, new technologies for PAD treatment have

\section{Corresponding author:}

Adam Janas, Center of Cardiovascular Research and Development, American Heart of Poland, 41 Czajek St, 40-534 Katowice, Poland, e-mail: adamjjanas@gmail.com

Received: 31.01.2019, accepted: 11.08.2019. 
emerged, including pharmacological regimens, endovascular and open surgery and stem cell therapy [3]. Nevertheless, the most frequent treatment for severe claudication and critical limb ischemia remains endovascular procedures and one of the most intensively developed technologies is atherectomy [4]. The main difference between the two devices compared in this study is the plaque modification mechanism. In rotational atherectomy the cutting blades are placed on the tip of the device, whereas in the directional one the cutting blades are placed on the side of the catheter. The asymmetrical rotational crown coated with microdiamonds is used in orbital atherectomy. Some devices use ultrasound waves for plaque modifications [4]. Novel devices are equipped with an intravascular imaging system such as optical coherence tomography (OCT) [5] or they use laser light to debulk the lesion [6]. The two atherectomy devices available on the market and used most frequently are the directional and rotational ones. Nonetheless, there is a lack of direct comparison between these two types of atherectomy in PAD.

\section{Aim}

Therefore, the aim of the study was to compare longterm outcomes after PAD endovascular revascularization performed with two types of atherectomy devices - rotational (AR) (Phoenix Philips) and directional (AD) (SilverHawk Medtronic).

\section{Material and methods}

\section{Subjects}

The paper is based on a retrospective study of 182 consecutive patients with symptomatic PAD who underwent endovascular revascularization with directional (AD) or rotational atherectomy (AR) between 2010 and 2015 at the San Antonio Endovascular \& Heart Institute. Adult patients ( $>18$ years old) with both intermittent claudication (Rutherford $3^{\text {rd }}$ class) and critical limb ischemia (CLI) (Rutherford $4^{\text {th }}-6^{\text {th }}$ class) were included if they had at least 1 lesion with $>70 \%$ diameter stenosis confirmed on the live quantitative vessel angiography in a lower extremity artery. Patients with in-stent restenosis were excluded.

\section{Procedural characteristic and pharmacological regimen}

Directional (Silver Hawk, Medtronic) and rotational (Phoenix Philips) atherectomy (AT: atherectomy) devices were applied in this study. The choice of the AT type was left to the operator's discretion. Atherectomy was followed by low-pressure balloon post-dilatation if residual stenosis was $>30 \%$. A distal protection system was not used for any patients. Angiographic success was defined as post-procedural TIMI 3 flow, no dissection and residual stenosis $<30 \%$. If angiographic success was not achieved, bail-out stenting was performed. Aspirin ( $81 \mathrm{mg} /$ day) was continued indefinitely whereas clopidogrel ( $75 \mathrm{mg} /$ day) was advised to be continued for 12 months after the procedure, as well as atorvastatin at the maximum tolerable dose, usually $40 \mathrm{mg}$ daily.

\section{Atherectomy devices}

The Silver Hawk (Medtronic) plaque excision system is a forward cutting directional atherectomy device. This can be used with or without concurrent percutaneous balloon angioplasty and stenting. The device consists of a rotating blade inside a tubular housing with a collection area nosecone. This catheter is connected to a battery-driven motor which spins the cutter.

The device comes in various sizes to enable atherectomy in vessels with diameters of $1.5-7.0 \mathrm{~mm}$. The advantage of atherectomy performed with the Silver Hawk device is the directional control, which makes it easier to remove eccentric lesions. As the device is advanced through the lesion, plaque is excised and packed in the nosecone. Different planes of excision are achieved by rotation of the device. Distal embolization remains a major disadvantage with these systems and hence the use of embolic protection devices is recommended in large and heavily calcified vessels [7].

The Phoenix Atherectomy System has a minimum working length of $130 \mathrm{~cm}$. The inward-cutting helical blade sits within a housing that acts as a shield and has an open area to expose the cutter. The cutter is rotated at high speeds (10,000 to $12,000 \mathrm{rpm})$ and shaves material directly into the housing. The debulked material is then conveyed to the proximal part of the catheter by an Archimedes screw on the outside of the torque shaft, which continuously conveys the excised plaque through the handle and into a collection reservoir outside the patient. The tip of the largest available catheter $(2.4 \mathrm{~mm})$ can be deflected to various degrees and rotated so that the cutter can debulk eccentrically to a larger diameter than the catheter itself. The controls for deflection and rotation are housed in the catheter handle with a self-contained battery-powered motor, and the operation of the Phoenix does not require any capital equipment components. This atherectomy can be used in vessels with diameter ranging from 2.0 to $7.0 \mathrm{~mm}$.

Both devices are approved for use in atherectomy of the peripheral vasculature and are not approved for use in coronary, carotid, iliac, or renal arteries [8].

\section{Study endpoints and definitions}

Because of the observational nature of this study, no preliminary hypothesis was generated. Target lesion revascularization (TLR) was considered the primary endpoint and was defined as any symptom-driven revascular- 
ization within a previously treated segment. Unplanned amputation related to a previously treated vessel, death and change in Rutherford class were regarded as secondary endpoints. Moreover, incidents of vessel perforation, dissection, distal embolization, and bailout stenting were collected.

\section{Safety and ethics}

This retrospective study was conducted in accordance with standard ethics guidelines. Endovascular procedures were carried out by experienced interventional cardiovascular teams in a high-volume center with a vascular surgery back-up within 30 min of transportation.

Owing to the observational and retrospective nature of this study, neither the patients' consent nor ethics committee approval was required.

\section{Data collection and follow-up}

Clinical and procedural data were collected on case report forms generated by the hospital electronic system, containing all patient hospitalization and discharge information. This system is audited for institutional quality assurance by private insurance companies and the state health fund.

The long-term follow-up data were collected during ambulatory check-ups or by telephone. The follow-up office visits were usually scheduled each 3-5 months. Some patients had phone visits due to lack of symptoms; nevertheless an office-based follow-up was scheduled on a later date. All outcomes of interest were confirmed using hospital discharge charts. Three patients met exclusion criteria for in-stent restenosis and one patient was lost to follow-up.

\section{Statistical analysis}

Continuous variables are presented as mean \pm SD or median (IQR). Data were compared using the $t$-test for parametric or Mann-Whitney $U$-test for non-parametric continuous variables. Categorical variables are reported as frequencies (percentages) and were compared using the Fisher exact test. Survival curves were constructed using the Kaplan-Meier estimates and were compared using the log-rank test. All reported $p$-values are 2-tailed, and $p<0.05$ was considered significant. GraphPad 6 Prism was used for the statistical analysis.

\section{Results}

The baseline characteristic of both groups is shown in Table I. There were no significant differences between the groups in baseline demographics except a higher rate of critical limb ischemia in the AR group. The mean follow-up for AD and AR was $282.6 \pm 147.4$ and 255.7 \pm 186.1 days, respectively $(p=0.44)$.

Lesions characteristics were similar between groups with the mean number of lesions equaling 1.5 and 1.4 per $A R$ and $A D$ patient, respectively. In both groups the primary lesion location was the anterior tibial artery. Furthermore, there were no significant differences between the groups in terms of lesion morphology in the TransAtlantic Inter-Society Consensus (TASC) and the number of chronic total occlusion. The procedural characteristics are shown in Table II. The number of periprocedural complications was low and similar between groups (Table III). The TLR was significantly more frequent in the AD group than in AR (AD: 25 (29.0\%) vs. AR: 15 (15.9\%) $p=0.03$ ) (Figure 1). There were no significant differences in the

Table I. Demographic characteristics

\begin{tabular}{|c|c|c|c|}
\hline Parameter & Directional & Rotational & $P$-value \\
\hline$N$ & 85 & 97 & \\
\hline Male, \% (n) & $58(49)$ & $59(57)$ & 0.88 \\
\hline Age, mean $\pm S D$ & $70.8 \pm 13.27$ & $73.1 \pm 9.8$ & 0.18 \\
\hline Hypertension, \% (n) & $98.8(84)$ & $97.8(95)$ & 1.0 \\
\hline Diabetes, \% (n) & $61(52)$ & $67(65)$ & 0.44 \\
\hline Hyperlipidemia, \% (n) & $95(81)$ & $96.9(95)$ & 0.42 \\
\hline $\mathrm{CAD}, \%(n)$ & $75.2(64)$ & $72(76.5)$ & 1.0 \\
\hline Post CABG, \% (n) & $27.3(23)$ & $97.7(19)$ & 0.29 \\
\hline Post PCI, \% (n) & $53.1(41)$ & $48.8(51)$ & 0.65 \\
\hline Post PAD revascularization, $\%(n)$ & $3.5(3)$ & $4.1(4)$ & 1.0 \\
\hline Dialysis reliant, \% (n) & $15(17.6)$ & $19.5(19)$ & 0.84 \\
\hline CLI, \% (n) & $20(23.5)$ & $50.5(49)$ & 0.02 \\
\hline
\end{tabular}

$C A D$ - coronary artery disease, $C A B G$ - coronary artery bypass graft, $P C I$ - percutaneous coronary intervention, $P A D$ - peripheral artery disease, $C L I$ - critical limb ischemia. 
Table II. Procedural characteristics

\begin{tabular}{|c|c|c|c|}
\hline Characteristics & Directional & Rotational & $P$-value \\
\hline Revascularized arteries $(n)$ & 117 & 143 & \\
\hline Superficial femoral artery, \% ( $n$ ) & $13(16)$ & $12(18)$ & 0.85 \\
\hline Profunda femoral artery, \% ( $n$ ) & $1(1)$ & $1(1)$ & 1.0 \\
\hline Popliteal artery, \% (n) & $11(13)$ & $10(15)$ & 1.0 \\
\hline Graft, \% (n) & $1(1)$ & $1(2)$ & 1.0 \\
\hline Anterior tibial artery, \% ( $n$ ) & $34(40)$ & $32(47)$ & 1.0 \\
\hline Trunk, \% (n) & $10(12)$ & $11(16)$ & 0.84 \\
\hline Posterior tibial artery, \% (n) & $19(22)$ & $20(28)$ & 0.87 \\
\hline Peroneal artery, \% ( $n$ ) & $10(12)$ & $10(15)$ & 1.0 \\
\hline Dorsalis pedis artery, \% ( $n)$ & $1(1)$ & $1(1)$ & 1.0 \\
\hline ATK, \% $(n)$ & $25(30)$ & $25(36)$ & 1.0 \\
\hline $\mathrm{BTK}, \%(n)$ & $75(87)$ & $75(107)$ & 1.0 \\
\hline CTO, \% $(n)$ & $14(17)$ & $16(23)$ & 0.86 \\
\hline TASC A, \% $(n)$ & $20(6)$ & $20(7)$ & 1.0 \\
\hline TASC B, \% $(n)$ & $37(11)$ & $36(13)$ & 1.0 \\
\hline TASC C, \% $(n)$ & $33(10)$ & $34(12)$ & 1.0 \\
\hline TASC D, \% $(n)$ & $10(3)$ & $10(4)$ & 1.0 \\
\hline
\end{tabular}

ATK - above the knee, BTK - below the knee, CTO - chronic total occlusion, TASC - TransAtlantic Inter-Society Consensus.

Table III. Periprocedural complications

\begin{tabular}{lccc} 
Complication & Directional & Rotational & $P$-value \\
\hline Artery perforation, \% $(n)$ & $1.2(1)$ & $2.0(2)$ & 1.0 \\
\hline Flow limiting dissection, \% $(n)$ & $2.0(2)$ & $3.1(3)$ & 1.0 \\
\hline Distal embolization, \% $(n)$ & 0 & 0 & 1.0 \\
\hline Bailout stenting, \% $(n)$ & $2.3(2)$ & $3.2(3)$ & 1.0
\end{tabular}

rate of death (AD: $1(1.7 \%)$ vs. AR: $5(5.7 \%) ; p=0.54)$ or amputations (AD: 2 (2.3\%) vs. AR: 5 (5.7\%); $p=0.45)$. There were no significant differences in time to TLR (Figure 2), amputation or death rate between the groups. There were no significant differences in the Rutherford class between the groups during follow-up. However, comparing before and after revascularization assessment there was a significant drop in the Rutherford class in both groups $(<0.0001)$, as shown in Figure 3.

\section{Discussion}

This study presents the single center outcomes of patients revascularized with the two types of atherectomy: directional and rotational. According to the available literature, the present study describes for the first time long-term outcomes of revascularization with two types of atherectomy in claudication and critical limb ischemia

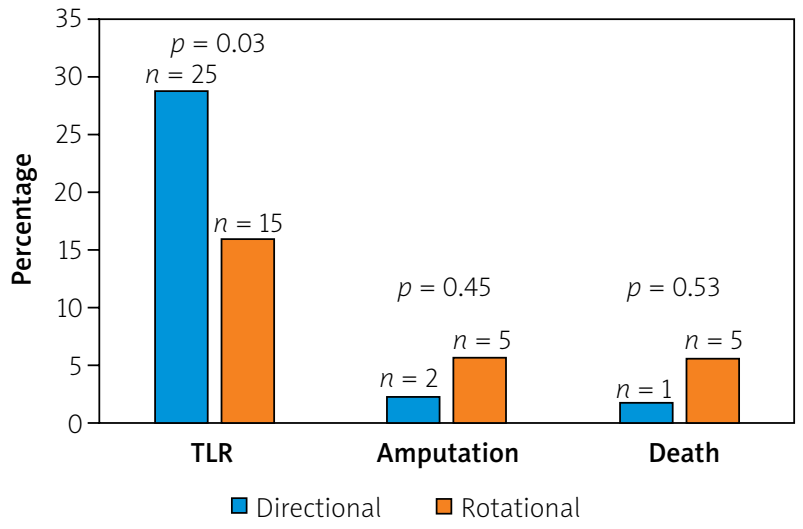

Figure 1. Target lesion revascularization, amputation, death

$T R L$-target lesion revascularization. 


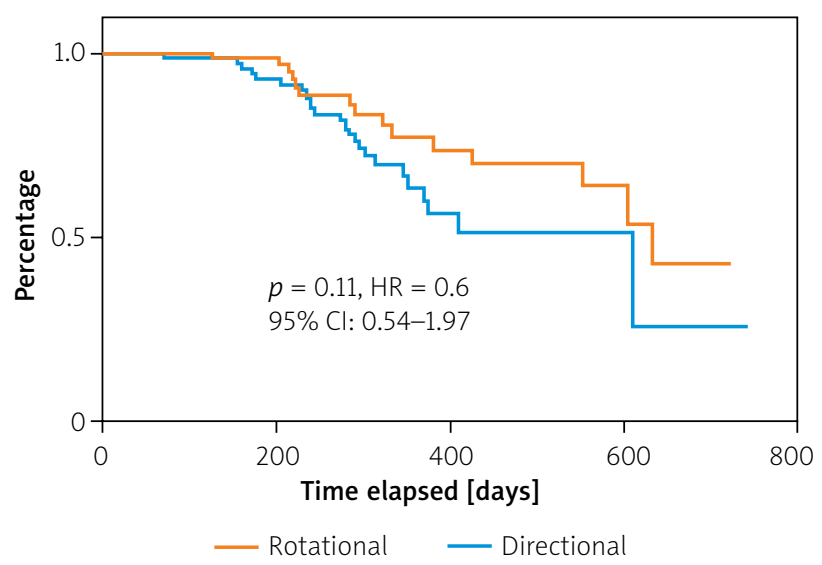

Figure 2. Kaplan-Meier curve showing target lesion revascularization free survival time

patients. The demographics were comparable between the groups with the exception of the CLI rate, being in favor of the AD group. Nevertheless, the procedural characteristics show no differences between the groups. The periprocedural complications, death and amputation were comparable between the groups. However, the TLR rate differed significantly in favor of the AR group.

To the best of our knowledge this is the first comparison of the two types of atherectomy used in PAD revascularization. In the EASE study the outcomes of PAD treatment with the Phenix Philips atherectomy device were comparable to the results we achieved. The rate of periprocedural complications was low and comparable (perforation - $5 \%$, dissection - $2 \%$, distal embolization - 1\%). However, in the 6-month follow-up the TLR rate was $12.4 \%$ and patients with CLI were excluded from the study [8]. The second rotational atherectomy device available on the market is the Pathway Jetstream Boston Scientific. In the published studies the periprocedural complications were more frequent than in our study. The authors reported $9 \%$ of dissections and $10 \%$ of minor embolization. The perforation scale was similar. Total rate of TLR after 12 months of follow-up was $27.1 \%$, which is higher than in our study [9]. In the other study the outcomes of PAD treatment with the Pathway Jetstream Boston Scientific were compared between patients with and without diabetes mellitus (DM). The TLR rates after 12 months of follow-up were $20 \%$ and $28 \%$ in DM and non-DM patients, respectively [10]. There is an abundance of reports on the PAD treatment with directional atherectomy. In the TALON study, SilverHawk was used to treat PAD in 601 patients. The rate of TRL after a 6-and 12-month follow-up was $10 \%$ and $20 \%$, respectively [11]. In the LA-DEFINITIVE study, where 800 subjects with both claudication and CLI were revascularized with directional atherectomy (SilverHawk/TurboHawk Medtronic), the primary patency rate was $81.5 \%$ [12].

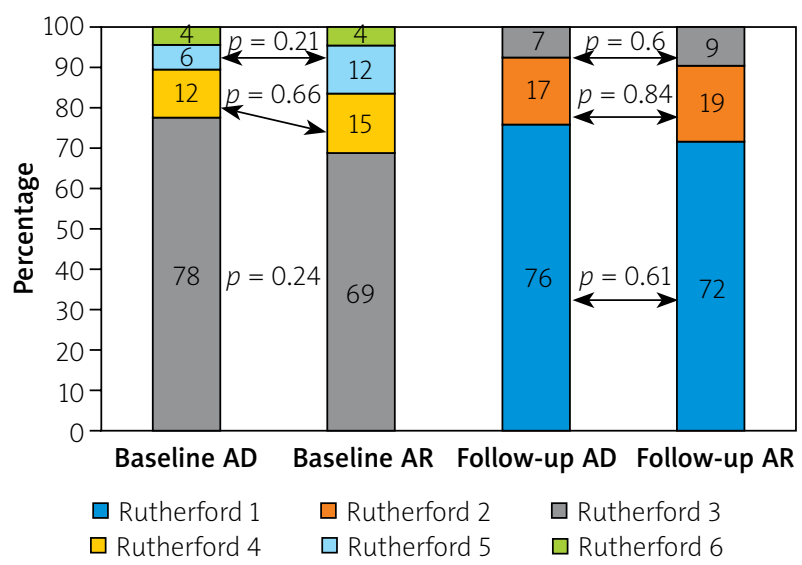

Figure 3. Rutherford classification prior and after treatment

The unification of indications, contraindications, atherectomy device choice and technique of debulking procedure, according to peer-reviewed expert opinion based on trials and registries could improve outcomes after revascularization with atherectomy in PAD, as has already happened in the coronary field [13].

A novel technology that may by combined with atherectomy in PAD is local drug delivery after revascularization. Early reports on the combination of plaque modification with atherectomy and subsequent DEB seem to be promising $[14,15]$. Nevertheless, a meta-analysis published recently by Katsanos et al. showed that the relative risk of all-cause mortality increases after the use of paclitaxel-coated device in PAD treatment in 2 and 5 years by $68 \%$ and $93 \%$, respectively [16]. Novel technologies, including local drug delivery nanotechnology, may soon become available for the follow-up treatment of plaque modifications after atherectomy [17]. Another interesting technology is directional atherectomy combined with optical coherence tomography, which allows more efficient debulking of the lesion while sparing adventitia. This technology is used in the Pantheris catheter, the safety and efficiency of which were confirmed in the Vision study [5].

To summarize, this study shows that the outcomes of the application of the rotational atherectomy in PAD are better than in the case of the directional device. This fact generates a hypothesis that $A D$ could injure the vessel wall more deeply than AR. Nonetheless, it should be confirmed in future studies where the AT revascularization is followed by intravascular imaging techniques, such as optical coherence tomography (OCT) or intravascular ultrasound (IVUS). Despite encouraging results of lower extremities' artery revascularization with atherectomy, still plain old balloon angioplasty and stenting is recommended for invasive treatment $[18,19]$. Moreover, laser ablation is no better than mechanical debulking with atherectomy. The only confirmed recommendation for endovascular laser ablation is in stent restenosis [20]. 
The main drawbacks of this analysis are those inherent to any single-center observational study [21] along with the difference of the CLI rate between the groups. The exact data on the very long below-the-knee chronic total occlusion are unavailable. The ankle brachial index $(A B I)$, ultrasonography doppler and toe pressure were not performed at each visit, making these data unsuitable for statistical analysis. During the study only one type of atherectomy device was available in the study site at the same time. This study is hypothesis generating only.

\section{Conclusions}

In this hypothesis-generating study of patients with symptomatic $P A D$, revascularization with the rotational atherectomy had a lower rate of TLR when compared to the directional one, despite the increased $C L I$ rate in the AR group. The periprocedural complications, amputations and death rate were comparable. Nevertheless, this should be confirmed in further controlled randomized trials.

\section{Conflict of interest}

The authors declare no conflict of interest.

\section{References}

1. Fowkes FG, Aboyans V, Fowkes FJ, et al. Peripheral artery disease: epidemiology and global perspectives. Nat Rev Cardiol 2017; 14: 156-70.

2. Selvin E, Erlinger TP. Prevalence of and risk factors for peripheral arterial disease in the United States. Circulation 2004; 110: 738-43.

3. Suzuki J, Shimamura M, Suda $H$, et al. Current therapies and investigational drugs for peripheral arterial disease. Hypertens Res 2016; 39: 183-91.

4. Bhat TM, Afari ME, Garcia LA. Atherectomy in peripheral artery disease: a review. J Invasive Cardiol 2017; 29: 135-44.

5. Schwindt AG, Bennett JG Jr, Crowder WH, et al. Lower extremity revascularization using optical coherence tomography-guided directional atherectomy: final results of the eValuatlon of the pantheriS optlcal cOherence tomography imagiNg atherectomy system for use in the peripheral vasculature (VISION) study. J Endovasc Ther 2017; 24: 355-66.

6. Mallios A, Blebea J, Buster B, et al. Laser atherectomy for the treatment of peripheral arterial disease. Ann Vasc Surg 2017; 44: 269-76.

7. Akkus NI, Abdulbaki A, Jimenez E, et al. Atherectomy devices: technology update. Med Devices (Auckl) 2015; 8: 1-10.

8. Davis T, Ramaiah V, Niazi K, et al. Safety and effectiveness of the Phoenix Atherectomy System in lower extremity arteries: early and midterm outcomes from the prospective multicenter EASE study. Vascular 2017; 25: 563-75.

9. Zeller T, Krankenberg H, Steinkamp H, et al. One-year outcome of percutaneous rotational atherectomy with aspiration in infrainguinal peripheral arterial occlusive disease: the multicenter pathway PVD trial. J Endovasc Ther 2009; 16: 653-62.

10. Sixt S, Scheinert D, Rastan A, et al. One-year outcome after percutaneous rotational and aspiration atherectomy in infraingui- nal arteries in patient with and without type 2 diabetes mellitus. Ann Vasc Surg 2011; 25: 520-9.

11. Ramaiah V, Gammon R, Kiesz S, et al. Midterm outcomes from the TALON Registry: treating peripherals with SilverHawk: outcomes collection. J Endovasc Ther 2006; 13: 592-602.

12. McKinsey JF, Zeller T, Rocha-Singh KJ, et al. Lower extremity revascularization using directional atherectomy: 12-month prospective results of the DEFINITIVE LE study. JACC Cardiovasc Interv 2014; 7: 923-33.

13. Dobrzycki S, Reczuch K, Legutko J, et al. Rotational atherectomy in everyday clinical practice. Association of Cardiovascular Interventions of the Polish Society of Cardiology (Asocjacja Interwencji Sercowo-Naczyniowych Polskiego Towarzystwa Kardiologicznego - AISN PTK): expert opinion. Kardiol Pol 2018; 76: 1576-84.

14. Stavroulakis K, Bisdas T, Torsello G, et al. Combined directional atherectomy and drug-eluting balloon angioplasty for isolated popliteal artery lesions in patients with peripheral artery disease. J Endovasc Ther 2015; 22: 847-52.

15. Tellez A, Dattilo R, Mustapha JA, et al. Biological effect of orbital atherectomy and adjunctive paclitaxel-coated balloon therapy on vascular healing and drug retention: early experimental insights into the familial hypercholesterolaemic swine model of femoral artery stenosis. Eurolntervention 2014; 10: 1002-8.

16. Katsanos K, Spiliopoulos S, Kitrou P, et al. Risk of death following application of paclitaxel-coated balloons and stents in the femoropopliteal artery of the leg: a systematic review and meta-analysis of randomized controlled trials. J Am Heart Assoc 2018; 7: e011245.

17. Granada JF, Tellez A, Baumbach WR, et al. In vivo delivery and long-term tissue retention of nano-encapsulated sirolimus using a novel porous balloon angioplasty system. Eurolntervention 2016; 12: 740-7.

18. Aboyans V, Ricco JB, Bartelink ML, et al. 2017 ESC guidelines on the diagnosis and treatment of peripheral arterial diseases, in collaboration with the European Society for Vascular Surgery (ESVS). Eur Heart J 2018; 39: 763-816.

19. Ruzsa Z, Wojtasik-Bakalarz J, Nyerges A, et al. S. Long-term follow-up after retrograde recanalization of superficial femoral artery chronic total occlusion. J Invasive Cardiol 2017; 29: 336-9.

20. Katsanos K, Spiliopoulos S, Reppas L,et al. Debulking atherectomy in the peripheral arteries: is there a role and what is the evidence? Cardiovasc Intervent Radiol 2017; 40: 964-77.

21. Biondi-Zoccai GG, Agostoni P, Abbate A. Parallel hierarchy of scientific studies in cardiovascular medicine. Ital Heart J 2003; 4: 819-20. 